\title{
A Novel Dynamic Bandwidth Allocation Mechanism for Star-Ring-Based EPON
}

\author{
I-Shyan Hwang, ${ }^{1}$ Jhong-Yue Lee, ${ }^{1}$ and Zen-Der Shyu ${ }^{2}$ \\ ${ }^{1}$ Department of Computer Science and Engineering, Yuan-Ze University, Chung-Li 32003, Taiwan \\ ${ }^{2}$ Department of General Studies, Army Academy, Chung-Li 32092, Taiwan
}

Correspondence should be addressed to I-Shyan Hwang, ishwang@saturn.yzu.edu.tw

Received 8 March 2011; Accepted 10 April 2011

Academic Editor: H. Shalaby

Copyright (C) 2011 I-Shyan Hwang et al. This is an open access article distributed under the Creative Commons Attribution License, which permits unrestricted use, distribution, and reproduction in any medium, provided the original work is properly cited.

\begin{abstract}
Ethernet Passive Optical Network (EPON) is proposed as a simple, cost-effective and scalable solution for bandwidth bottlenecks in access networks which can enhance the system performance because it transmits the aggregated high-speed traffic from hundreds of subscribers. Dynamic Bandwidth Allocation (DBA) mechanism has not yet been seriously considered for use in star-ring EPON architecture. In this paper, a distributed QoS-based dynamic bandwidth allocation (DQ-DBA) scheduling mechanism for the star-ring-based EPON architecture including optical line terminal (OLT), Sub-OLT and ONUs has been proposed to improve the system performance of traditional tree-based EPON architecture. In DQ-DBA, the highest-priority traffic of each ONU is directed to the OLT by the tree structure; moreover, the lower-priority traffic on each ONU is transmitted to the Sub-OLT by ring architecture. This approach can effectively resolve the idle period problem and reduce the overloading of OLT in conventional DBA mechanisms. Exhaustive simulation experiments are performed to compare the system performance between the tree and the star-ring architectures and validate the effectiveness of the proposed mechanism. Simulation results show that the proposed DQ-DBA mechanism in star-ring architecture can reduce packet delay and jitter for the high-priority traffic, thus ensuring the quality of service (QoS) regardless of subscriber numbers.
\end{abstract}

\section{Introduction}

Ethernet Passive Optical Network (EPON), standardized by the IEEE 802.3ah [1] Ethernet in the First Mile (EFM) task force, shows the promise for overcoming bandwidth blockages in access networks. The tree-based EPON architecture, shown in Figure 1, consists of two main components: the optical line terminal (OLT) residing at the central office (CO) and the multiple optical network units (ONUs) located at the customer residence area. Ethernet frames are transmitted by the OLT, passing through a $1: N$ passive splitter to reach each ONU. The passive splitter is generally located far from the $\mathrm{CO}$, but close to the ONUs at the customer residence area.

The EPON comprises both upstream and downstream data transmissions. In the downstream direction, the OLT broadcasts frames to all ONUs; and the upstream direction is a multipoint-to-point (MP2P) network through which only single ONU may transmit data in its time slot to avoid signal collisions. The IEEE 802.3ah task force has defined some control messages through the development of the multipoint control protocol (MPCP). The two primary messages are the REPORT message for upstream transmission and the GATE message for downstream transmission. The ONU sends the bandwidth requests to OLT by the REPORT messages. After the OLT receives these messages from the ONUs, the OLT then sends a GATE message to each ONU within the limits of the time to begin transmission and the size of the assigned time slot.

Categorizing traffics into different classes is essential for guaranteeing quality of service (QoS) for differentiated services in EPONs. To ensure QoS, EPONs support the principal IP-based differentiated services (DiffServ) mechanism [2]. For instance, the highest-priority data can be mapped to expedited forwarding (EF) [3] which provides time-critical, low-loss, and bandwidth-guaranteed services which typically require a constant bit rate (CBR), such as voice transmission. 


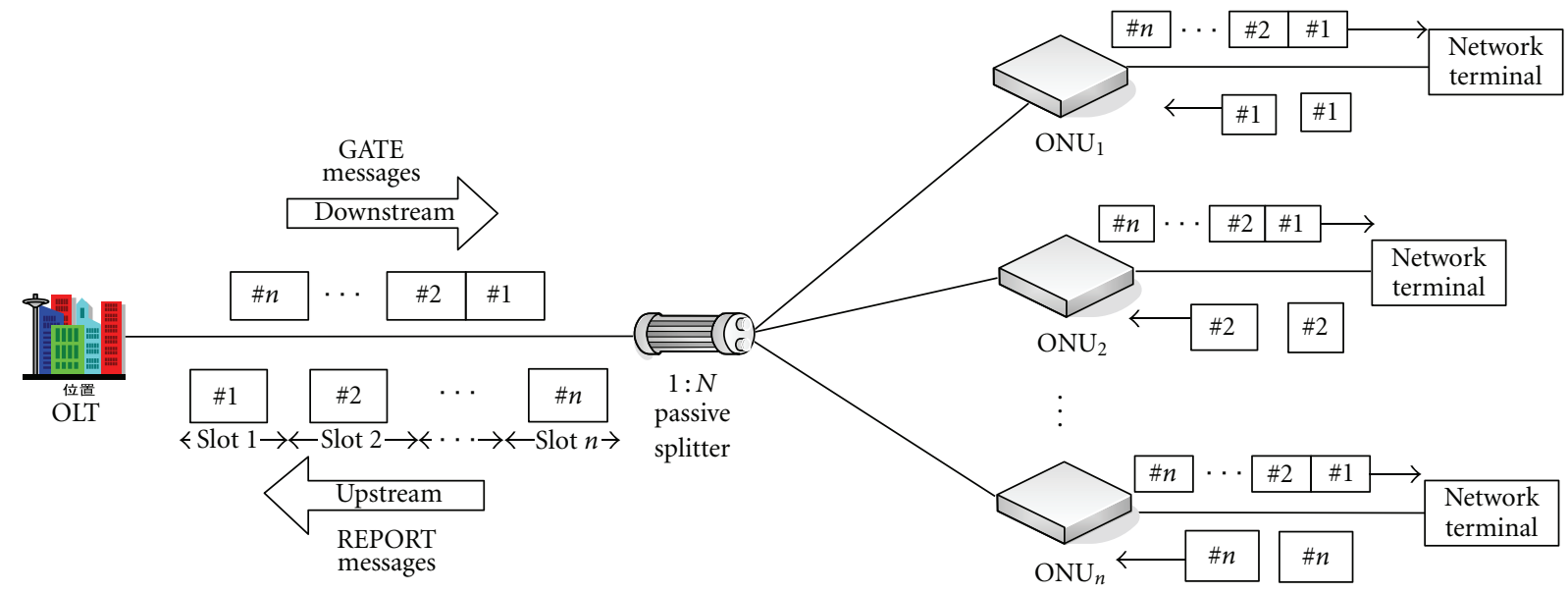

Figure 1: Tree-based PON topology.

The medium-priority data can be mapped to assured forwarding (AF), which is intended for services that are not delay-sensitive but require bandwidth guarantees, which is typical of variable bit rate (VBR) services, such as video streaming. Finally, the low-priority data can be mapped to best effort (BE), which is neither delay-sensitive nor bandwidth-guaranteed, and is appropriate for applications such as web browsing, background file transfer, and e-mail. $\mathrm{AF}$ and $\mathrm{BE}$ traffics are more delay tolerant but are generally wideband. EF traffic is very delay sensitive but tends to be narrowband. A fixed and properly sized cycle length with a fixed EF traffic can provide reduced delays and jitter-free operation. In EPONs, the carried traffic is greatly influenced by the scheduling discipline which plays an important role in ensuring network performance through the equitable allocation of available network resources. A scheduling discipline includes two principal functions: first, it decides the order in which requests are serviced; second, it manages the request service queue. Designing an efficient algorithm for OLT bandwidth assignment is a critical issue for EPONs.

In traditional dynamic bandwidth allocation (DBA) [4], each ONU sends a REPORT message to the OLT at the end of an assigned transmission time slot. The OLT collects the REPORT messages from each ONU, and then sends a GATE message back to each ONU for the next cycle. The time during which the ONUs are waiting for the OLT's instructions is called the idle period-the sum of computation time of DBA and round-trip times between the OLT and each ONU. Reducing the idle period can improve bandwidth utilization and enhance system performance. Moreover, traditional DBA scheduling algorithms have some drawbacks, such as excessive OLT overhead and guard time, resulting in wasted bandwidth. For the DBAs in [5-7], the OLT dynamically allocates a variable timeslot to each ONU based on immediate bandwidth conditions, and ensures the QoS by providing a guaranteed service level agreement (SLA) to efficiently improve the system performance.

PON architectures are commercially deployed as tree topologies, providing flexibility and simplicity in the deployment of access networks. Recently, the tree-based EPON architecture has been extended and enhanced for some specific objectives. Shami et al. proposed a cascaded twostage EPON architecture [8], termed Sub-OLT, which adds a new intermediate level of ONUs to the network to reduce OLT overhead, as shown in Figure 2(a). Sub-OLT raises the possibility of further dividing an ONU's capacity amongst multiple lower-level bandwidth users via an evenly branched PON setup. Therefore, the Sub-OLT functions in a hybridized manner and assembles the functionality of back-to-back pairs of connected ONU and OLT nodes, as illustrated in Figure 2(b). This architecture not only helps increase the viability of optical access over a broader range of subscriber access scenarios, but also performs well in the calculation of an extra stage, which significantly reduces the OLT hardware challenges. However, the addition of the SubOLT presents an additional cost.

Ring-based PON designs offer several additional features, such as support of truly shared local area network (LAN) capabilities among end users as well as simple and costeffective protection against failures in the distribution network. Figure 3(a) illustrates a generic view of a distributed ring-based EPON architecture proposed by Pathak et al. [9]. This architecture supports heterogeneous traffics of wired and wireless services and makes use of the time division multiplexing (TDM) and potentially wavelength division multiplexing (WDM) PON approaches. At each ONU, the downstream and LAN traffic is first demultiplexed by the WDM coupler, shown in Figure 3(b), and the LAN traffic is received and processed at each ONU. The ONU generates signals which are then transmitted to the next ONU, allowing the ONUs to independently and sequentially run instances of the DBA algorithm. Moreover, it presents an error-free ring architecture which allows the signal to bypass a failed ONU on to the next ONU and terminate at the OLT. Thus, the LAN transmission delay is equal to zero. However, this paper still has a drawback in that it only considers that failure might occur in the nodes but not in the links.

In the ITU-T Recommendation G.983.1, four types of network protection architectures are proposed $[10,11]$. The first type only protects the link between the OLT and the 


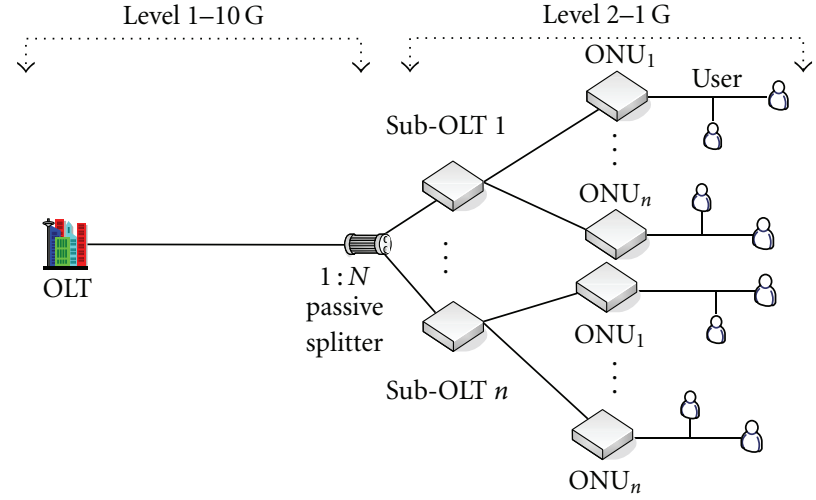

(a) Architecture

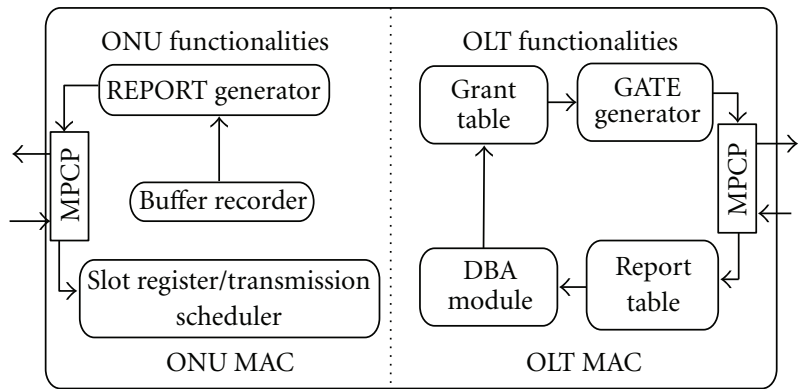

(b) Sub-OLT functionalities

Figure 2: Two-stage EPON.

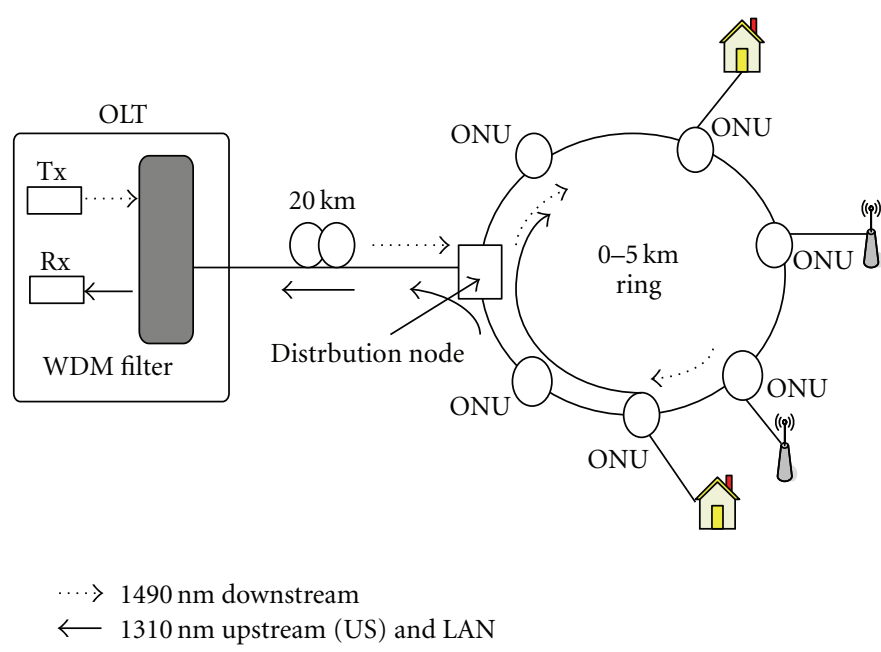

(a) Architecture $\longleftarrow$ Downstream

২... Upstream
ONU architecture and operation

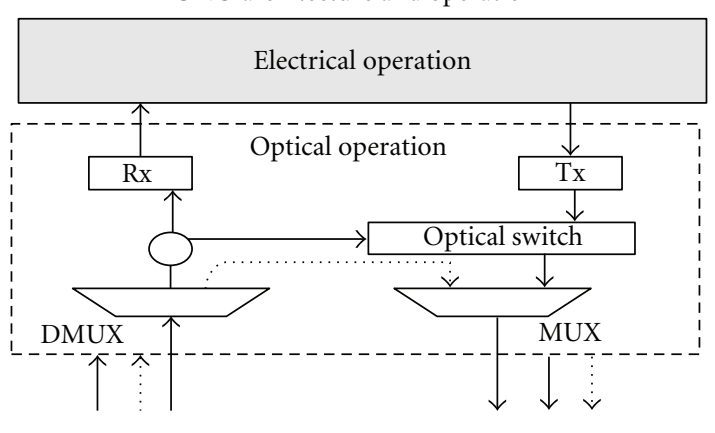

(b) ONU operation

Figure 3: Ring-based EPON.

splitter and is of no use if the failure occurs in either the OLT or the splitter. The second type improves on the first type by building a back-up OLT module. The third type provides a comprehensive protection for all the links and nodes and ensures that data is simultaneously sent to the working link and the protection link (similar to $1+1$ protection), providing the network with full protection. However, the installation costs for this approach are high and it wastes resources through ineffective load sharing between the backup nodes and the protection link. The fourth type improves on the third type by adding one splitter and one protection link in EPON. However, in this approach, the backup nodes and protection links are unable to share the burden of the major nodes and links, and this approach also has the highest installation cost of all four types.

Aside from ITU-T protection schemes, Sun et al. proposed a star-ring EPON architecture [12], shown in Figure 4, to provide protection against fiber link failure between the remote node (RN) and the ONUs, thus rendering the OLT transparent to such fiber failure. This architecture provides excellent protection against the fiber link failure. However, no DBA mechanism has been proposed on this architecture to date.

This paper proposes a star-ring architecture, shown in Figure 5(a), including one OLT, one Sub-OLT (designated from one of the ONUs and playing the role of a gatherer in the LAN), and ONUs. A distributed QoS-based dynamic bandwidth allocation (DQ-DBA) scheduling mechanism is proposed in star-ring architecture which transmits highpriority traffic to the OLT through traditional tree architecture to guarantee low-delay jitter performance, and the ring architecture transmits lower-priority traffic to the Sub-OLT to reduce guard time between ONUs and share computing overhead in the PON system. This star-ring architecture allows the proposed DQ-DBA mechanism to allocate bandwidth requests in such a way as to improve propagation delay performance and reduce idle periods without degrading QoS support for other service types. To formularize this DQ-DBA mechanism, some parameter definitions are summarized in Table 1. 


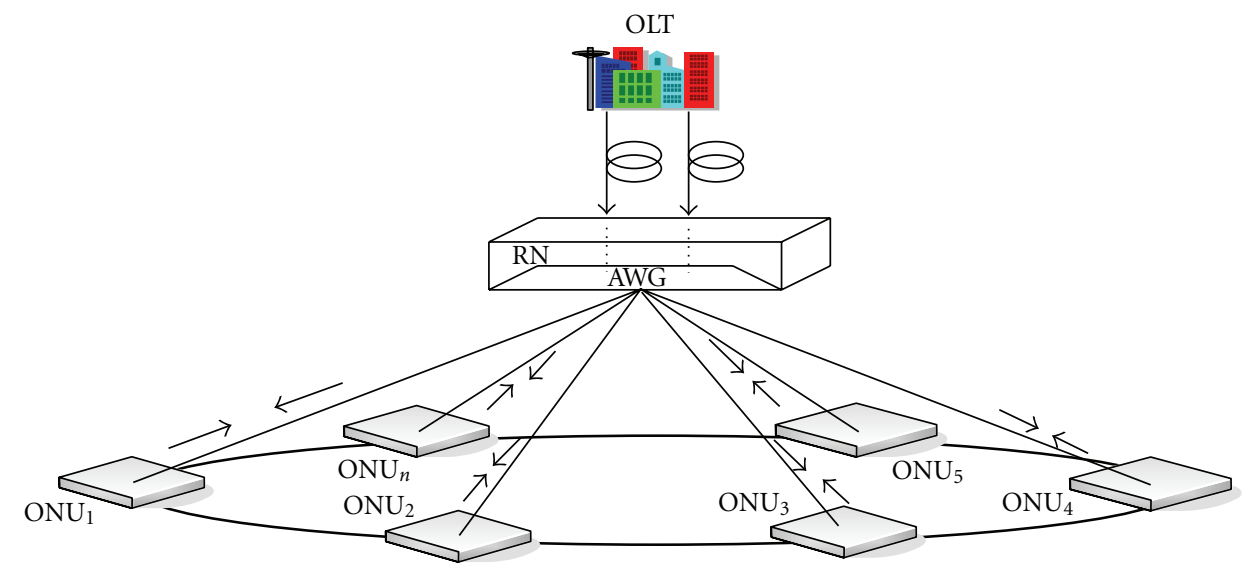

FIGURE 4: Star-ring architecture.

TABLE 1: Parameter definitions.

\begin{tabular}{ll}
\hline$T_{\text {cycle }}$ & Maximum time in each cycle \\
$B_{\text {available }}$ & Available bandwidth in cycle time \\
$B_{\text {remain }}$ & Remaining available bandwidth \\
$R_{i}^{\mathrm{EF}}$ & EF bandwidth request of $\mathrm{ONU}_{i}$ \\
$R_{\mathrm{FIFO}}^{\mathrm{AF}}$ & AF bandwidth request in FIFO Queue \\
$R_{\mathrm{FIFO}}^{\mathrm{BE}}$ & BE bandwidth request in FIFO Queue \\
$C_{\text {capacity }}$ & Link capacity \\
$G_{i, n+1}^{\mathrm{EF}}$ & EF bandwidth granted of $\mathrm{ONU}_{i}$ in cycle $n$ \\
$G_{\mathrm{FIFO}}^{\mathrm{AF}}$ & +1 \\
$G_{\mathrm{FIFO}}^{\mathrm{BE}}$ & AF bandwidth granted in FIFO Queue \\
\hline
\end{tabular}

The rest of this paper is organized as follows. Section 2 proposes a distributed QoS-based DBA scheduling mechanism in a star-ring architecture. Section 3 describes simulation experiments conducted to study and compare the performance of the proposed DQ-DBA scheduling mechanism in star-ring-based architecture with static bandwidth allocation (SBA) and the IPACT algorithm [6] in a tree-based architecture. The final conclusions are drawn in Section 4.

\section{Star-Ring Architecture and DQ-DBA Mechanism}

2.1. Star-Ring Architecture. In this paper, we propose a distributed QoS-based DBA (DQ-DBA) scheduling mechanism based on the star-ring architecture for resolving the idle period problem, and reducing EF jitter and OLT overhead. As shown in Figure 5(a), the combination network topology can be visualized as a three-dimensional star-ring structure consisting of three main components-the OLT, which is similar to traditional OLTs; the Sub-OLT, which is designated by one of the ONUs; and the Sub-OLT scheduling function as illustrated in Figure 5(b). The Sub-OLT includes two levels of scheduling. In the first level, the scheduling is based on FIFO to collect the AF queue and BE queue for DiffServ-aware traffic. The main function of second level is to allocate bandwidth based on the SLA. Therefore, the Sub-OLT scheduling will sequentially satisfy the bandwidth requests from the $\mathrm{AF}$ and $\mathrm{BE}$ queues. In the proposed errorfree architecture, the signal will be bypassed each ONU to the next ONU and terminated at Sub-OLT according to the ring architecture. Unlike previous PON topologies, in this starring architecture, the OLT and ONUs are interconnected via a long fiber (tree structure) and ONUs are interconnected via a short distribution fiber ring in the local loop, but share the standard trunk feeder fiber for long reach connectivity to the OLT and distribution fiber to the Sub-OLT.

2.2. DQ-DBA Mechanism. In the distributed QoS-based DBA (DQ-DBA) operation, one ONU is selected as the SubOLT in the star-ring architecture. The highest-priority traffic (EF) of each ONU is sent to the OLT through the tree structure, and the lower-priority traffic (AF and BE) of each ONU is sent to the Sub-OLT through the ring architecture. Next, in the ring-based architecture, after the ONU combines the backlog with received data, it will send the whole data to the next ONU. Finally, the Sub-OLT will receive the upstream data from each ONU to process the Sub-OLT scheduling. On the OLT side, the DBA mechanism will comply with the SubOLT bandwidth request following the EF bandwidth request for each ONU and the Sub-OLT will provide a local DBA scheduling mechanism, decreasing the computing load of the OLT.

The DQ-DBA mechanism, shown in Figure 6, comprises two operations: at the beginning of each transmission cycle, each ONU sends its EF traffic and REPORT message to the OLT. After the Sub-OLT sends its REPORT message to the OLT, the OLT allocates bandwidth for each ONU. At this time, the Sub-OLT can send packets of AF, and BE traffic from each ONU to the OLT. Then, the OLT sends a GATE message to each ONU for the next cycle. The advantages of DQ-DBA mechanism are that it reduces the idle period, EF delay, EF jitter, and OLT overhead to improve the system performance of EPONs.

In the scheduling of the OLT, the transmission cycle time is the sum of the transmission time and guard times for all 


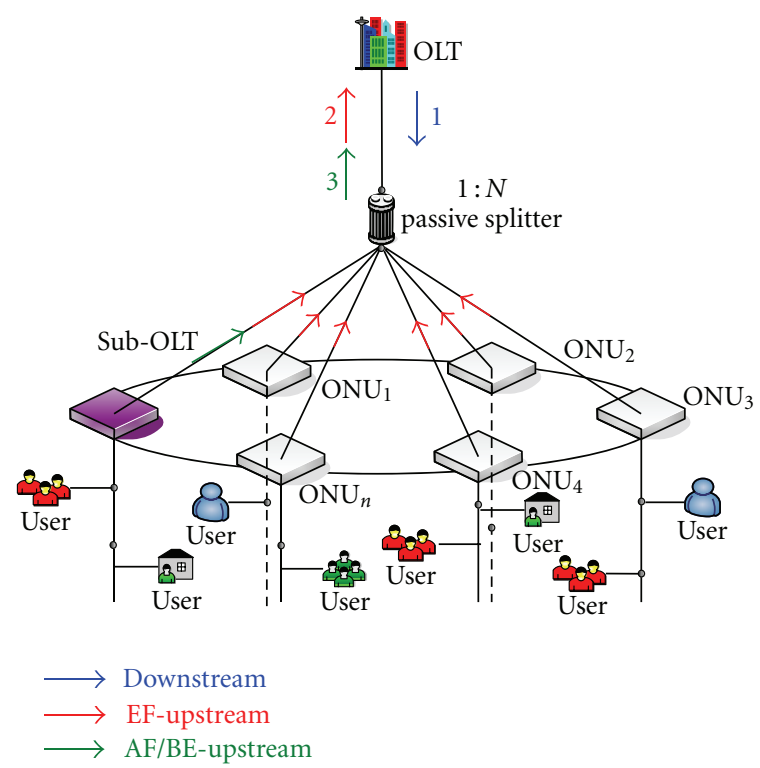

(a) Architecture

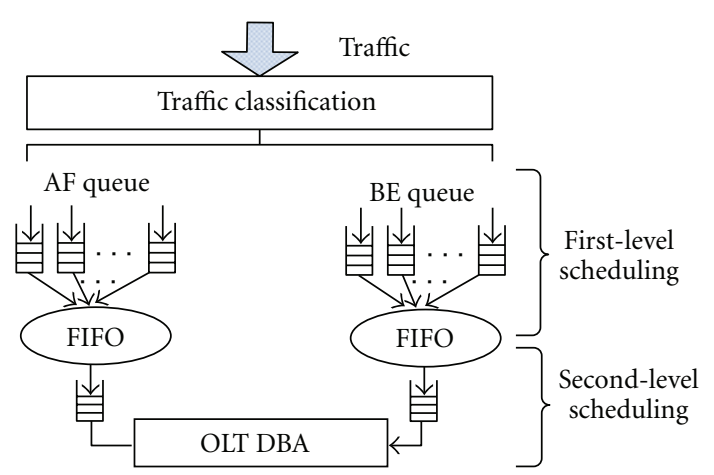

(b) Sub-OLT architecture

Figure 5: Star-ring EPON.

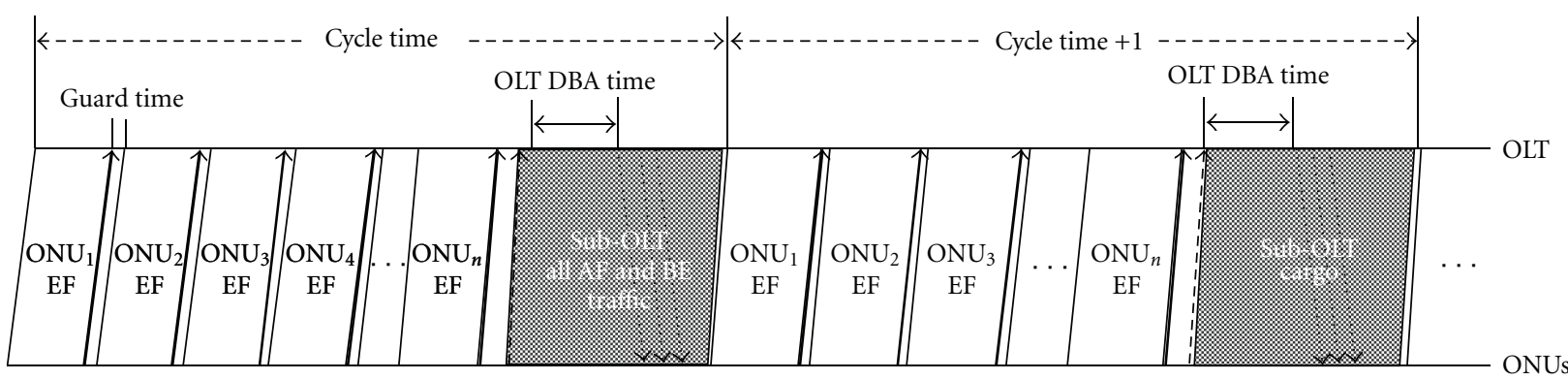

ONUs

\footnotetext{
$\longrightarrow$ EF REPORT message from each ONU

$-\rightarrow$ All AF and BE REPORT message from Sub-OTL

....> GATE message to ONU and Sub-OLT
}

FIgURE 6: Operation of the DQ-DBA mechanism.

ONUs. In one transmission cycle, we assume that each ONU can transmit a REPORT message to the OLT. The available bandwidth to upstream in one cycle, $B_{\text {available }}$, is expressed as (1)

$$
B_{\text {available }}=C_{\text {capacity }} \times\left(T_{\text {cycle }}-N \cdot g\right)-N \times 512,
$$

where $C_{\text {capacity }}$ is the OLT link capacity (bits/sec), $T_{\text {cycle }}$ is the maximum cycle time, $g$ is the guard time, and $N$ is the number of ONUs with a control message length of 512 bits (64 bytes).

The OLT DBA scheme is illustrated in Figure 7. At the outset, the OLT will gather EF request bandwidth of each ONU and then decides the grant bandwidth of EF traffic for the cycle $n+1, G_{i, n+1}^{\mathrm{EF}}$, based on SLA for each ONU when the sum of EF traffic bandwidth request, $\sum_{i=1}^{N} R_{i}^{\mathrm{EF}}$, is bigger than $B_{\text {available. The formula is illustrated as follows: }}$

$$
G_{i, n+1}^{\mathrm{EF}}=\frac{\mathrm{ONU}_{i}^{\mathrm{SLA}}}{\mathrm{SLA}_{\text {total }}} \times B_{\text {available }}
$$

Otherwise, the OLT will satisfy the EF bandwidth request of each ONU to avoid high priority traffic packet delay and follow by calculating the remaining available bandwidth, $B_{\text {remain }}$, for Sub-OLT schedule. The $B_{\text {remain }}$ can be expressed as follows:

$$
B_{\text {remain }}=B_{\text {available }}-\sum_{i=1}^{N} R_{i, n+1}^{\mathrm{EF}} .
$$

The Sub-OLT schedule is illustrated in Figure 8, with the Sub-OLT allocating bandwidth for the AF, and BE queues 


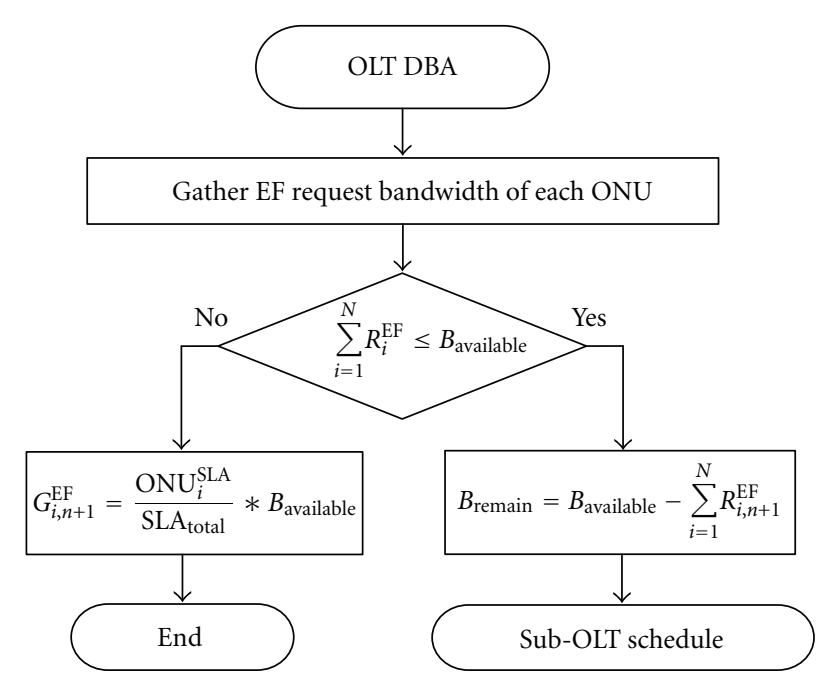

Figure 7: Flowchart of OLT DBA.

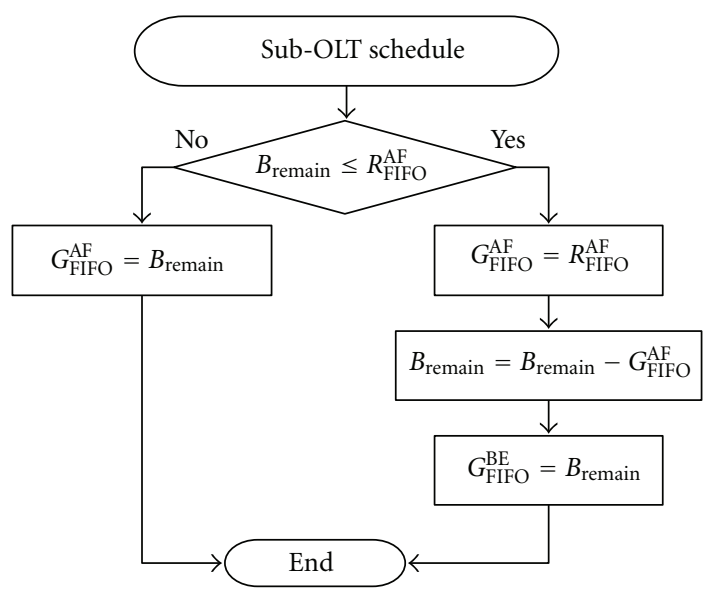

Figure 8: Flowchart of Sub-OLT.

according to the SLA. At the outset, the Sub-OLT compares the bandwidth request of the AF queue with the remaining bandwidth, $B_{\text {remain }}$. The FIFO queue of $\mathrm{AF}$ traffic, $R_{\mathrm{FIFO}}^{\mathrm{AF}}$, will be serviced first, followed by the FIFO queue of BE traffic, $R_{\mathrm{FIFO}}^{\mathrm{BE}}$. When the total request bandwidth of AF traffic is smaller than $B_{\text {remain }}$, the remaining bandwidth can be updated as $B_{\text {remain }}=B_{\text {remain }}-G_{\mathrm{FIFO}}^{\mathrm{AF}}$. On the other hand, the Sub-OLT just satisfies the request bandwidth of AF traffic when the total request bandwidth of AF traffic is bigger than $B_{\text {remain }}$.

\section{Performance Analysis}

This section compares the performance of the DQ-DBA mechanism in the star-ring architecture with static bandwidth allocation (SBA) and the IPACT algorithm [6] in tree architecture in terms of throughput, end-to-end delay, EF jitter, and blocking probability based on the SLA to ensure QoS. Performance is evaluated through the OPNET simulation tool. One wavelength channel is adopted with
TABle 2: Definition of parameters.

\begin{tabular}{ll}
\hline Number of ONUs & 32 \\
Number of wavelength & 1 \\
Upstream/downstream link capacity & $1 \mathrm{Gbps}$ \\
OLT-ONU distance (uniform) & $10-20 \mathrm{~km}$ \\
ONU-ONU distance (uniform) & $1 \mathrm{~km}$ \\
Maximum cycle time & $1 \mathrm{~ms}$ \\
Guard time & $5 \mu \mathrm{s}$ \\
Control message length & $0.512 \mu \mathrm{s}$ \\
Sub-OLT buffer Size & $10 \mathrm{MB}, 20 \mathrm{MB}$ \\
ONU buffer Size & $10 \mathrm{MB}$ \\
DBA computation time & $15 \mu \mathrm{s}$ \\
\hline
\end{tabular}

a link capacity of $1 \mathrm{~Gb} / \mathrm{s}$. The distance from one ONU to the OLT is assumed to be $10-20 \mathrm{~km}$, with a $10 \mathrm{MB}$ buffer for each ONU and in a first-in/first-out service policy for different buffer sizes of the Sub-OLT. Extensive study of the traffic model indicates that most network traffic can be characterized according to self-similarity and long-range dependence (LRD) [13]. This model is adopted to generate highly bursting $\mathrm{AF}$ and $\mathrm{BE}$ traffic classes with a Hurst parameter of 0.7. Packet sizes are uniformly distributed between 64 and 1518 bytes. In addition, the high-priority traffic (EF) is modeled on a Poisson distribution and the packet size is fixed to 70 bytes. To show the effectiveness of high-priority traffic, three different $\mathrm{EF}, \mathrm{AF}$, and $\mathrm{BE}$ traffic ratios are analyzed and compared: scenario_244 (20\%, 40\%, $40 \%)$, scenario_433 (40\%, 30\%, 30\%), and scenario_622 $(60 \%, 20 \%, 20 \%)[14,15]$. The simulation scenarios are summarized in Table 2.

3.1. Throughput. Figure 9 shows the system throughput of DQ-DBA in star-ring architecture and SBA (IPACT) in tree architecture with $10 \mathrm{MB}$ buffer size in each ONU and Sub-OLT for EF, AF, and BE traffic with different traffic ratio profiles for different traffic loads. Figure 9(a) shows that the proposed DQ-DBA mechanism outperforms SBA and IPACT in EF throughput, regardless of the traffic ratio profile. The reason is that EF traffic in the DQ-DBA mechanism is transmitted by the tree architecture which is always guaranteed at the outset of the transmission window in the proposed DQ-DBA mechanism. For the AF and BE throughputs, as shown in Figures $9(\mathrm{~b})$ and $9(\mathrm{c})$, the DQ-DBA mechanism is relatively similar to SBA until the traffic load exceeds $50 \%$, and IPACT until the traffic load exceeds $70 \%$, whereas the DQ-DBA has gradually achieved saturation performance until the traffic load of $70 \%$ because the $\mathrm{AF}$ and $\mathrm{BE}$ traffic will be satisfied after EF traffic transfer is complete. Thus, the QoS-based operation of DQ-DBA guarantees the requirements of high-priority services and pays less attention to the low-priority traffic. Moreover, given a Sub-OLT buffer size of $10 \mathrm{MB}$, low-priority services will be blocked in the Sub-OLT, resulting in lower AF and $\mathrm{BE}$ throughput than would be found in SBA and IPACT schemes. 


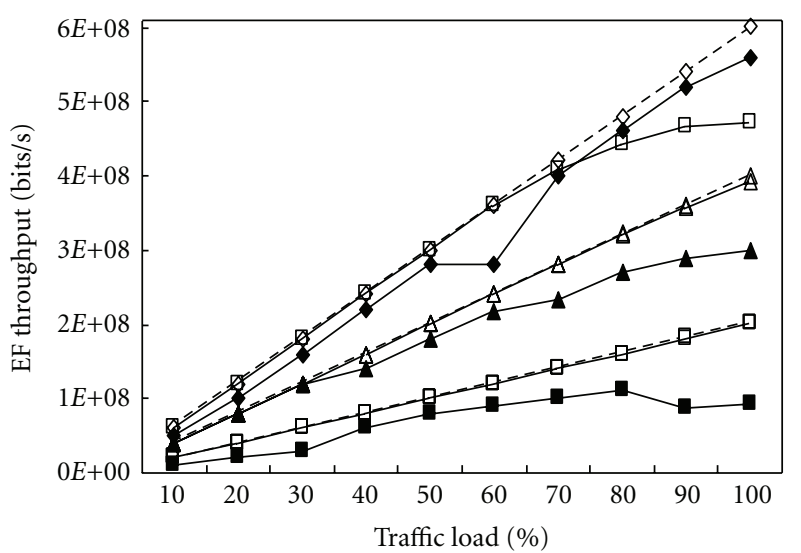

(a) EF throughput

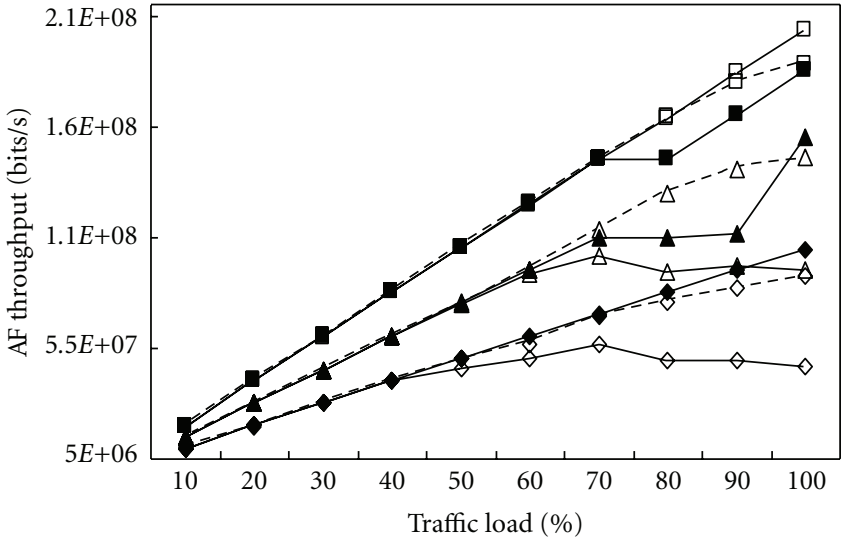

(b) AF throughput

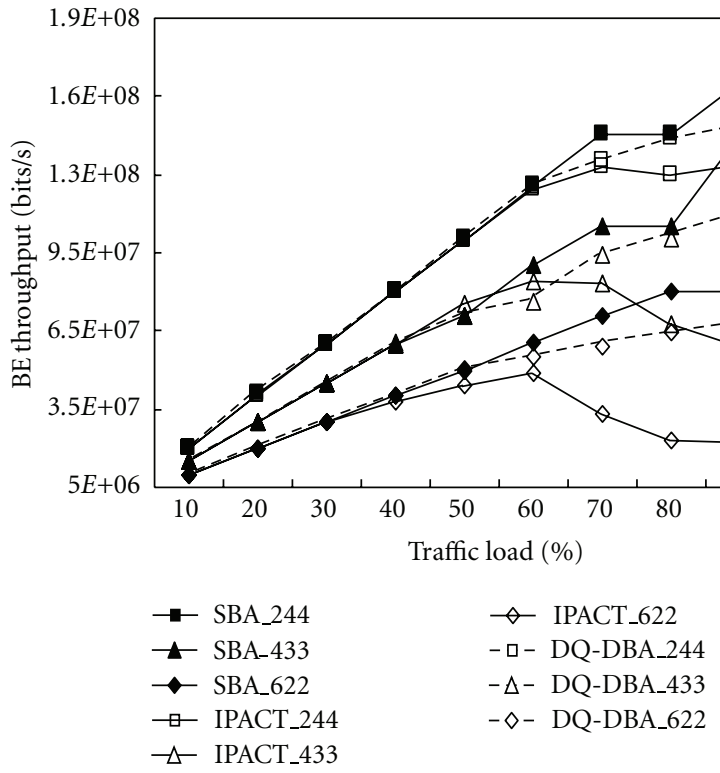

(c) BE throughput

Figure 9: Throughput comparisons with SBA, IPACT, and DQ-DBA.

3.2. End-to-End Delay. Figure 10 shows the end-to-end delay performance of the DQ-DBA in the star-ring architecture and SBA (IPACT) in the tree architecture with $10 \mathrm{MB}$ buffer size in ONU and Sub-OLT for EF, AF and BE traffic, with different traffic ratio profiles for different traffic loads. The end-to-end delay of EF and AF traffic in the proposed methods provide better QoS service than the SBA and IPACT for all traffic ratio profiles, as shown in Figures 10(a) and 10(b). However, the end-to-end delay of BE traffic in DQDBA is higher than IPACT, except in situations exceeding the $70 \%$ traffic load in scenario_433 and exceeding the $80 \%$ traffic load in scenario_622, as shown in Figure 10(c). The reason is that the DQ-DBA first transmits the AF traffic in the Sub-OLT to ensure the QoS, but will limit the BE end-toend delay by blocking packets in the $10 \mathrm{MB}$ buffer, resulting in higher, but not excessive, end-to-end delay.

3.3. EF Jitter. Figure 11 shows the EF jitter performance of the DQ-DBA in the star-ring architecture and SBA (IPACT) in the tree architecture with $10 \mathrm{MB}$ buffer size in each ONU and Sub-OLT for EF, AF and BE traffic with different traffic ratio profiles for different traffic loads. The delay variance $\sigma^{2}$ is calculated as $\sigma^{2}=\sum_{1}^{N}\left(d_{i}^{\mathrm{EF}}-\bar{d}\right)^{2} / N$, where $d_{i}^{\mathrm{EF}}$ is the delay time of the EF packet $i$ and $N$ is the total number of EF packets received [15]. Simulation results show that the delay variance for EF traffic increases as the traffic load increases in DQ-DBA. The proposed DQ-DBA mechanism outperforms the SBA and IPACT for EF jitter performance because the DQ-DBA sequentially transmits the whole EF traffic of every ONU at the outset of the transmission cycle time.

3.4. Blocking Probability. Figure 12(a) compares the blocking probability of DQ-DBA in the star-ring architecture and SBA (IPACT) in the tree architecture with $10 \mathrm{MB}$ buffer size in ONU and Sub-OLT, with different traffic ratio profiles for different traffic loads. Simulation results show that the blocking probability of the DQ-DBA algorithm is worse than that of IPACT because the bandwidth requests of $\mathrm{AF}$ and 


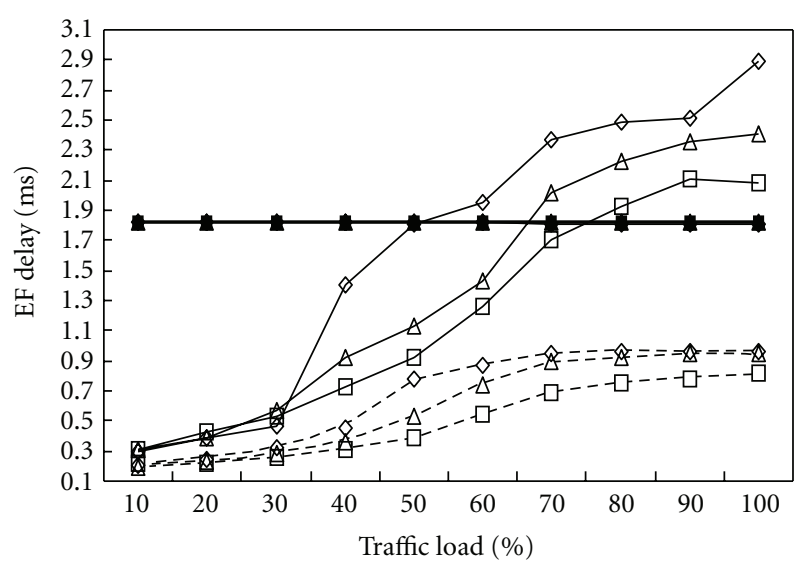

(a) EF end-to-end delay

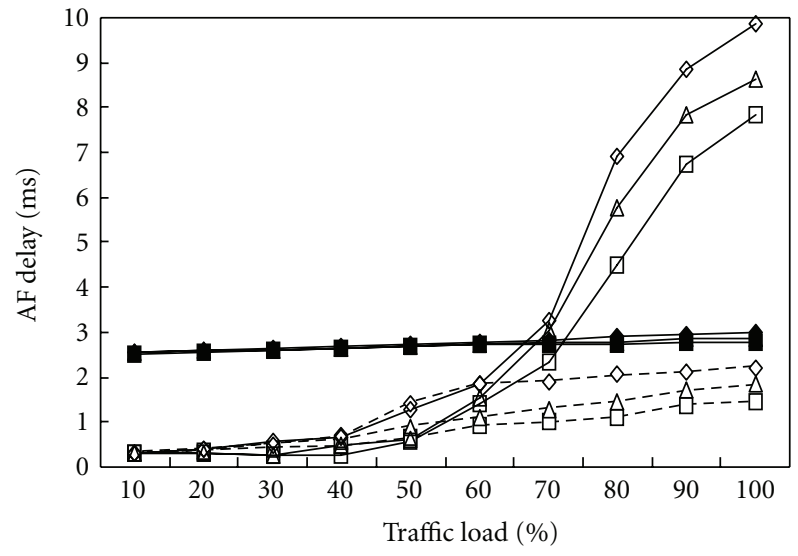

(b) AF end-to-end delay

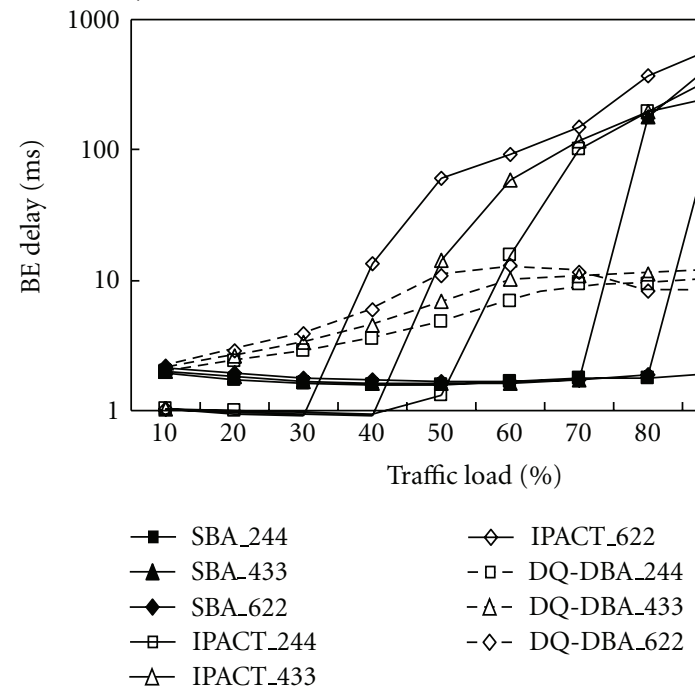

(c) BE end-to-end delay

FIGURE 10: End-to-end delay comparisons with SBA, IPACT, and DQ-DBA.

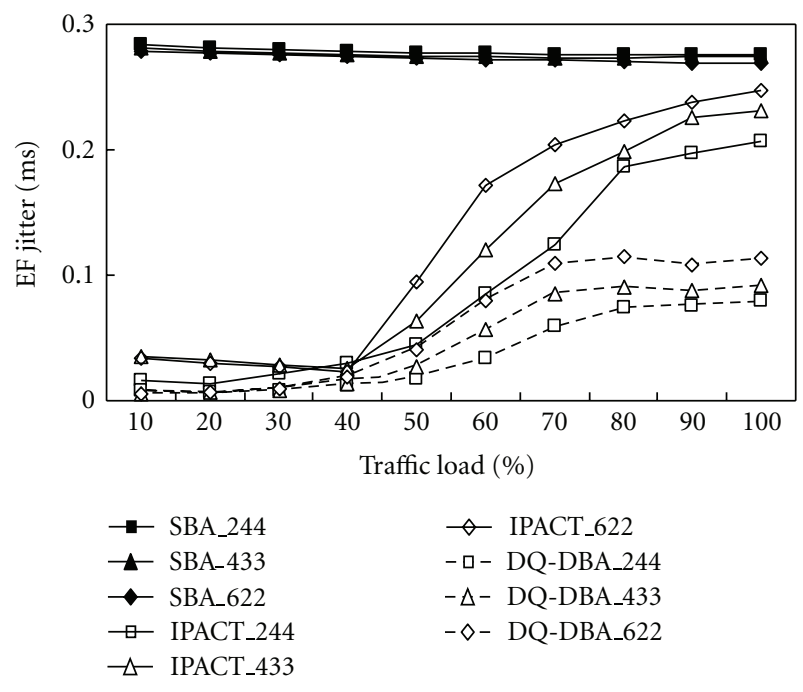

FIGURE 11: EF jitter comparisons with SBA, IPACT, and DQ-DBA.

BE traffic for every ONU must be sent to the Sub-OLT and the Sub-OLT cannot support such a large amount of services with a small buffer, thus resulting in a high blocking rate. Figure 12(b) compares the blocking probability of the DQDBA in the star-ring architecture with $10 \mathrm{MB}$ and $20 \mathrm{MB}$ buffer sizes in the Sub-OLT. It is worth noting that, in the proposed star-ring architecture, no blocking occurs with buffer sizes above $30 \mathrm{MB}$ for the 32 ONUs, regardless of traffic ratio profile.

\section{Conclusions}

In this paper, we proposed a DQ-DBA mechanism in a star-ring-based EPON architecture to reduce idle periods and improve EF delay and jitter performance. The DQDBA operates on a star-ring architecture ensuring the highest-priority traffic of each ONU is sent to the OLT by a tree structure, and the lower-priority traffic of each ONU are transmitted to Sub-OLT by a ring architecture. The advantages of DQ-DBA mechanism are that it reduces the idle period problem and overhead of the OLT, thus improving EPON system performance. Additionally, the DQ-DBA mechanism transmits EF traffic at the outset of the transmission window, which can also improve EF jitter 


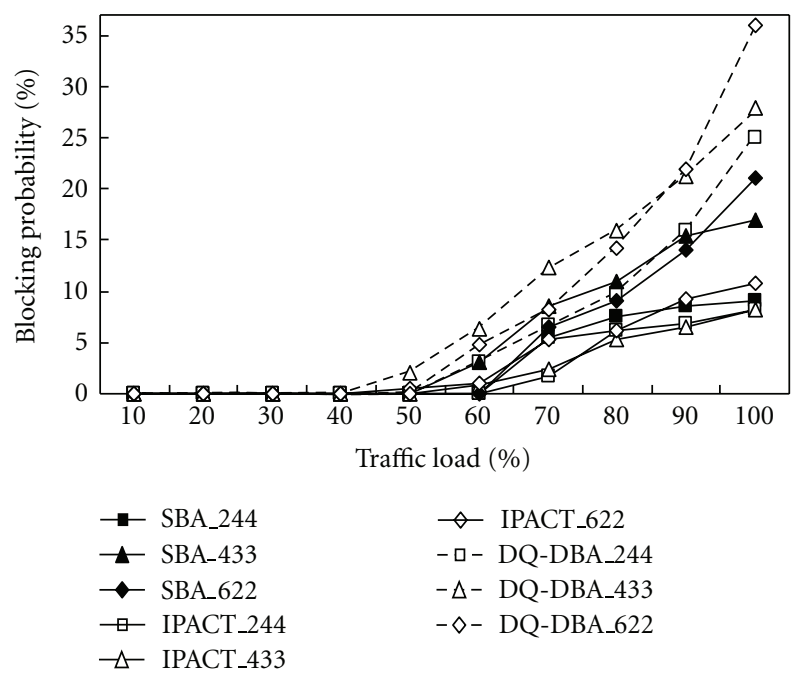

(a) Blocking probability of the SBA, IPACT and DQ-DBA with $10 \mathrm{MB}$ buffer size

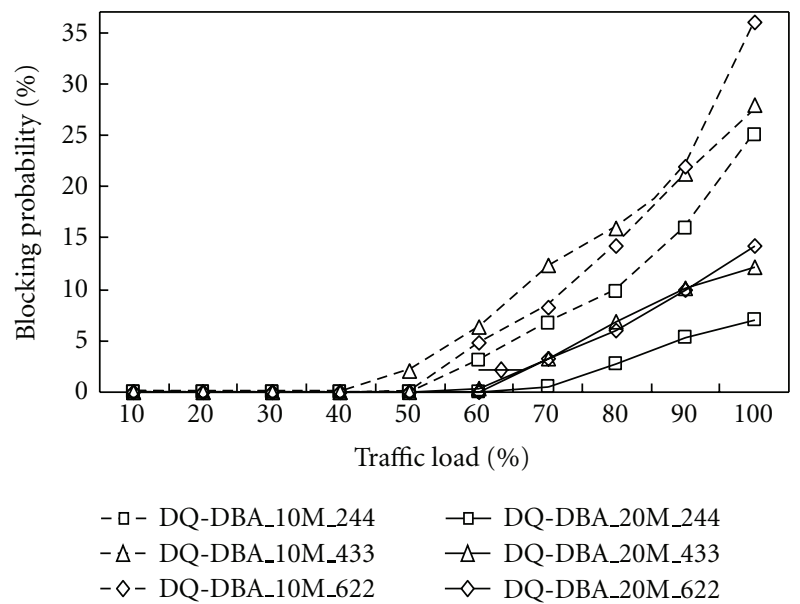

(b) Blocking probability of DQ-DBA with $10 \mathrm{MB}$ and $20 \mathrm{MB}$ buffer size

FIGURE 12: Blocking probability comparisons with SBA, IPACT, and DQ-DBA.

performance. Simulation results show that the proposed DQ-DBA mechanism can reduce packet delay and packet delay variation for high-priority ONUs to ensure QoS. For the end-to-end delay performance, the DQ-DBA can guarantee EF traffic delay below $1.1 \mathrm{~ms}$ in any scenario and also has excellent EF jitter performance. Future work will conduct more in-depth study into the prediction and excess bandwidth allocation mechanism for the OLT, the buffer size and handling mechanism for Sub-OLT, and the structure of fault-tolerant mechanism for star-ring architecture.

\section{References}

[1] IEEE P802.3ah Ethernet in the First Mile Task Force, http://www.ieee802.org/3/efm.

[2] S. Blake, D. Black, M. Carlson, E. Davies, Z. Wang, and W. Weiss, "An architecture for differentiated services," IETF RFC 2475, December 1998.
[3] V. Jacobson, K. Nichols, and K. Podur, "An expedited forwarding PHB," IETF RFC 2598, June 1999.

[4] S. De, V. Singh, H. M. Gupta, N. Saxena, and A. Roy, "A new predictive dynamic priority scheduling in Ethernet passive optical networks (EPONs)," Optical Switching and Networking, vol. 7, no. 4, pp. 215-223, 2010.

[5] N. A. M. Radzi, N. M. Din, M. H. Al-Mansoori, I. S. Mustafa, and S. K. Sadon, "Intelligent dynamic bandwidth allocation algorithm in upstream EPONs," Journal of Optical Communications and Networking, vol. 2, no. 1-3, pp. 148-158, 2010.

[6] G. Kramer, B. Mukherjee, and G. Pesavento, "Interleaved polling with adaptive cycle time (IPACT): a dynamic bandwidth distribution scheme in an optical access network," Photonic Network Communications, vol. 4, no. 1, pp. 89-107, 2002.

[7] Y. Luo and N. Ansari, "Bandwidth allocation for multiservice access on EPONs," IEEE Communications Magazine, vol. 43, no. 2, pp. S16-S21, 2005.

[8] A. Shami, X. Bai, N. Ghani, C. M. Assi, and H. T. Mouftah, "QoS control schemes for two-stage ethernet passive optical access networks," IEEE Journal on Selected Areas in Communications, vol. 23, no. 8, pp. 1467-1478, 2005.

[9] B. Pathak, M. A. Ummy, N. Madamopoulos, N. Antoniades, M. A. Ali, and R. Dorsinville, "Experimental demonstration of a distributed ring-based EPON architecture," Photonic Network Communications, vol. 19, no. 1, pp. 55-61, 2010.

[10] ITU-T, Study Group 15, "G.983: high speed optical access systems based on passive optical network (PON) techniques," 2001.

[11] X. L. Zhou, F. X. Yu, Y. C. Wen, and Z. M. Lu, "A novel protection architecture scheme for EPON," Information Technology Journal, vol. 10, no. 3, pp. 591-596, 2011.

[12] X. F. Sun, Z. X. Wang, C. K. Chan, and L. K. Chen, "A novel star-ring protection architecture scheme for WDM passive optical access networks," in Conference on Optical Fiber Communication, vol. 3 of Technical Digest Series, pp. 563-565, Anaheim, Calif, USA, March 2005.

[13] W. Willinger, M. S. Taqqu, and A. Erramilli, "A bibliographical guide to self-similar traffic and performance modeling for modern high-speed networks," in Stochastic Networks: Theory and Applications, Royal Statistical Society, vol. 4 of Lecture Notes Series, Oxford University Press, Oxford, UK, 1996.

[14] X. Bai and A. Shami, "Modeling self-similar traffic for network simulation," Tech. Rep. NetRep-2005-01, 2005.

[15] I. S. Hwang, Z. D. Shyu, C. C. Chang, and J. Y. Lee, "Faulttolerant architecture with dynamic wavelength and bandwidth allocation scheme in WDM-EPON," Photonic Network Communications, vol. 18, no. 2, pp. 160-173, 2009. 

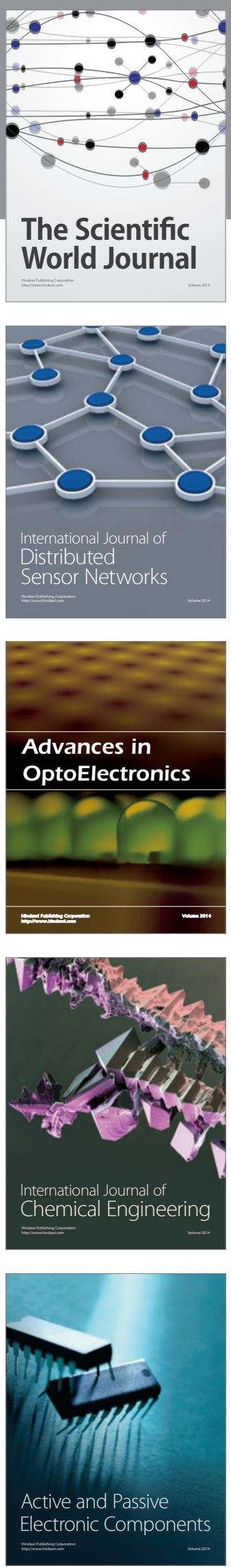
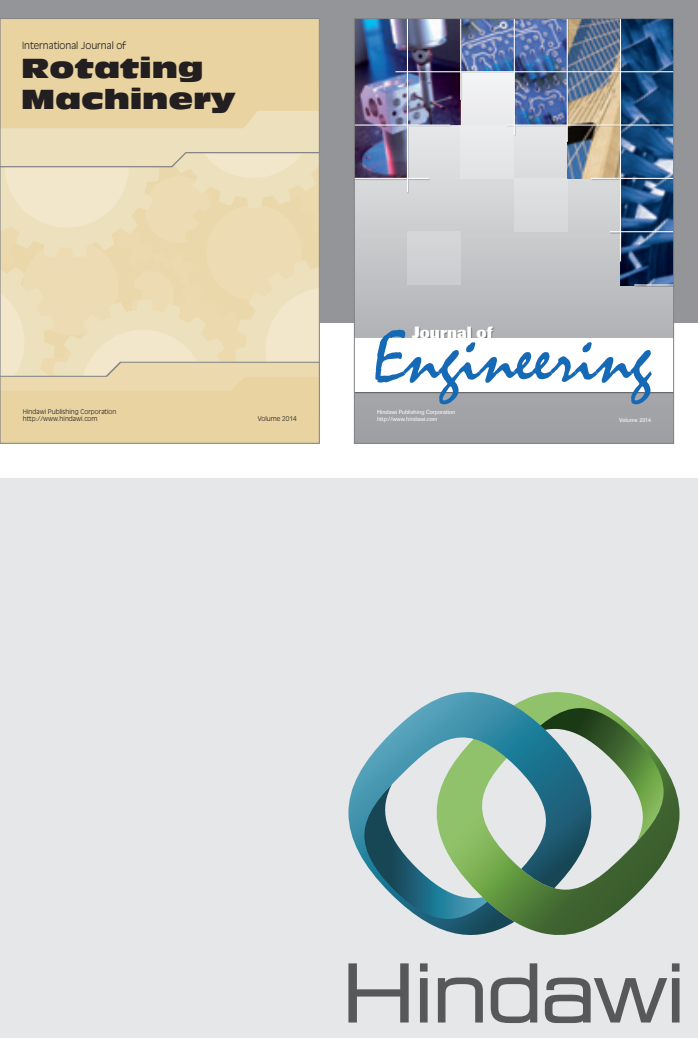

Submit your manuscripts at

http://www.hindawi.com
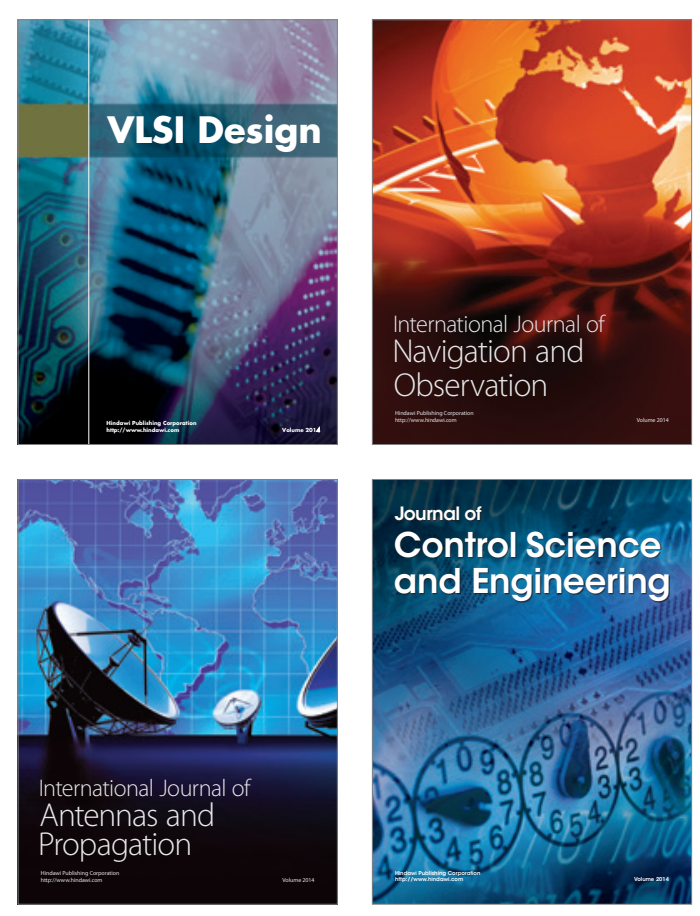
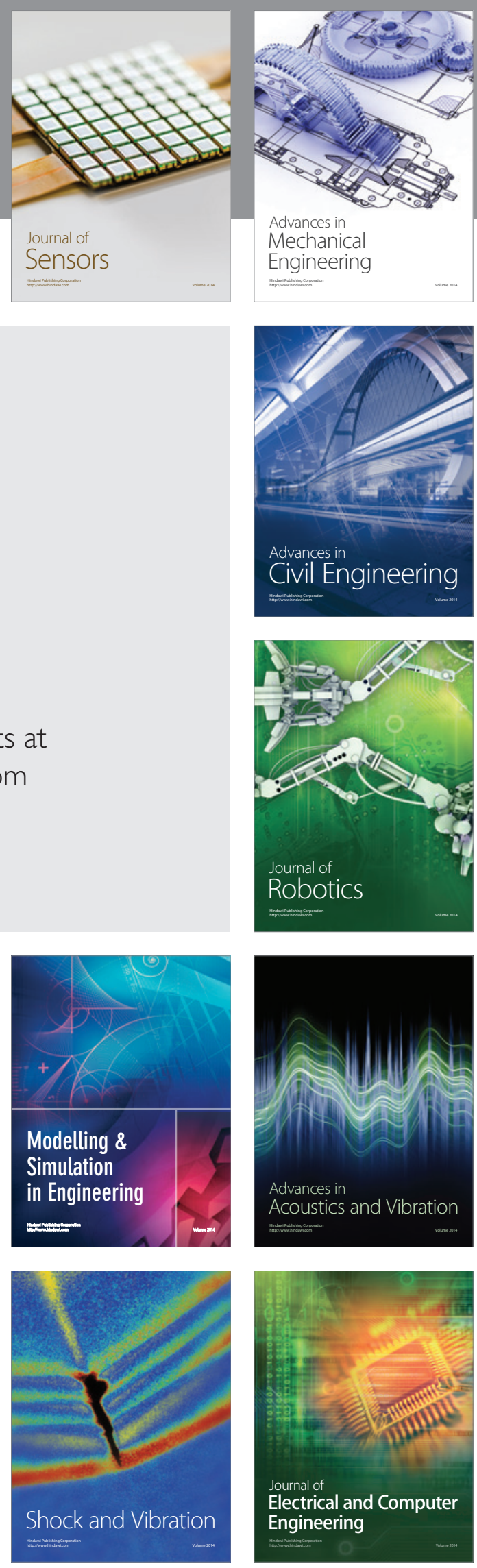